\title{
Influence of Dexmedetomidine on Toxicity of Intrathecal Ketamine on Neonatal Rat Spinal Function
}

\author{
Yue-Yi Ren ${ }^{1}$, Hai-Qing Zhang ${ }^{2}$, Shu-Hua Duan ${ }^{1}$ and Shi-Duan Wang ${ }^{3 *}$ \\ ${ }^{1}$ Heart Center, Qingdao Women and Children's Hospital, Qingdao University, Shandong -266011, ${ }^{2}$ Department of \\ Anaesthiology, Qingdao Women and Children's Hospital, Qingdao University, Shandong - 266011, ${ }^{3}$ Department of \\ Anaesthiology, The Affiliated Hospital of Qingdao University, Shandong - 266021, China
}

${ }^{*}$ For correspondence: Email: wangshiduan271@hotmail.com; Tel/Fax: 0086-532-82912515

\begin{abstract}
Purpose: To investigate the influence of dexmedetomidine on the effect of ketamine on developing spinal cord.

Methods: Postnatal day $3(P 3)$ and postnatal day 7 (P7) rat pups received intrathecal ketamine (10 $\mathrm{mg} / \mathrm{kg} \mathrm{b.wt)} \mathrm{and/or} \mathrm{dexmedetomidine} \mathrm{(10} \mu \mathrm{g} / \mathrm{kg}$ b.wt). Spinal reflex function was assessed by evaluating the sensory stimuli based on mechanical withdrawal threshold. Spinal tissue was analysed for activated caspase-3 using monoclonal anti-activated caspase-3. Apoptosis count of the spinal tissue was also measured by Fluoro-Jade $C$ staining while glial reactivity was assessed by ionized calcium binding adapter molecule 1 on Day 7 day following injection. Long-term spinal function in rat pups on postnatal day 35 (P35) was evaluated by measuring the hindlimb withdrawal threshold and gait analysis.

Results: Exposure to intrathecal ketamine at $10 \mathrm{mg} / \mathrm{kg}$ in $P 3$ and $P 7$ pups decreased mechanical withdrawal thresholds and increase apoptosis and microglial activation in the spinal cord. Altered spinal function, as presented by a decrease in mechanical withdrawal threshold and altered static gait, was observed in P35 rats exposed to intrathecal ketamine on P3. Dexmedetomidine administration did not alter the spinal function and histology of spinal tissue. Co-administration of dexmedetomidine and ketamine suggests that the former reduced apoptosis counts, altered glial responses, increased mechanical withdrawal threshold and improved gait.

Conclusion: Dexmedetomidine protects against intrathecal ketamine-induced spinal toxicity in neonatal rats.
\end{abstract}

Keywords: Dexmedetomidine, Intrathecal ketamine, Spinal toxicity, Mechanical withdrawal threshold, Glial reactivity, Gait analysis, Activated caspase-3, Apoptosis count

Tropical Journal of Pharmaceutical Research is indexed by Science Citation Index (SciSearch), Scopus, International Pharmaceutical Abstract, Chemical Abstracts, Embase, Index Copernicus, EBSCO, African Index Medicus, JournalSeek, Journal Citation Reports/Science Edition, Directory of Open Access Journals (DOAJ), African Journal Online, Bioline International, Open-J-Gate and Pharmacy Abstracts

\section{INTRODUCTION}

The effective use of spinal anaesthesia in neonates and infants has been well established in paediatric practice [1,2]. Series of studies have reported safe and effective use of anaesthesia and analgesia in paediatric practice [3]. In preterm neonates susceptible to post-operative apnoea or having co-existing respiratory disease neuraxial anaesthesia is largely used [4]. There is mounting evidence that the systemic general anaesthetics with n-methyl-D-aspartate (NMDA) antagonist or $y$-amino butyric acid agonist action can cause apoptosis of the developing brain cells in rodents [5]. The use of neuraxial anaesthesia and analgesia could avoid or minimize the exposure to general anaesthetics in neonates and infants. 
Non-competitive NMDA receptor antagonist, ketamine is widely used in paediatric anaesthesia to provide sedation and analgesia [6]. However, the developing brain is highly susceptible to anaesthesia. Repeated exposure to ketamine and other NMDA antagonists have been reported to cause neuroapoptosis in the developing brain of neonatal rats [7]. Other than systemic administration/route, ketamine is also administered spinally in combination with local anaesthetic to extend the duration and to improve the analgesic effect [8]. Spinal cord toxicity has been reported in ketamine-induced adult animals [9]. Intrathecal ketamine has been reported to induce spinal toxicity in neonatal rats [10].

Dexmedetomidine, a potent $\alpha 2$ - adrenoceptor agonist, has a broad safety margin and has excellent analgesic, sedative, and anaestheticsparing properties. It has also been reported to have neuroprotective effects in animal models [11]. Studies have demonstrated that dexmedetomidine reduced the required dose of ketamine and is also effective in attenuating the cardio-stimulatory and adverse effects of ketamine in non-human primates [12]. It remains unknown as to whether dexmedetomidine could possibly also attenuate ketamine-induced spinal toxicity. The aim of this study was to evaluate whether dexmedetomidine administration after intrathecal injection of ketamine can prevent ketamine-induced spinal toxicity in neonatal rats.

\section{EXPERIMENTAL}

All experiments were carried out according to protocols approved by the Institutional Animal Care and Use Committee of the Affiliated Hospital of Qingdao University, Shandong, China. Pregnant Holtzman Sprague-Dawley rats (Harlan, Indianapolis, IN) were housed in accordance with the guidelines for laboratory animals [13] in a $12 \mathrm{~h}$ light-dark cycle and provided with free access to standard feed and water. The litter day of the pups was noted. The pups were randomly assigned to treatment groups containing equal numbers ( $n=10 /$ group) of males and females at postnatal day 3 (P3) or day 7 (P7). Body weights of the rat pups were between $8-11 \mathrm{~g}$ at P3 and $12-18 \mathrm{~g}$ at P7. The animals were regularly monitored and maintained until further testing at 5 weeks of age. For all experimental interventions, rat pups were kept on heating pad $\left(36{ }^{\circ} \mathrm{C}-37^{\circ} \mathrm{C}\right)$ in order to maintain body temperature. Proper care was taken to minimize the duration of maternal separation and handling of the pups as well.
All chemicals used in the study were purchased from Sigma Aldrich, MO, USA, unless otherwise specified.

\section{Intrathecal injection}

Pups were anesthetized with isoflurane (3 - $5 \%$ ) in oxygen and air. Percutaneous intrathecal injections were made at the low lumbar level (intervertebral space L4-5 or L5-L6) with a 30gauge needle perpendicular to the skin. Injection volumes of $0.5 \mu \mathrm{l} / \mathrm{g}$ bodyweight (previously determined) [14], were delivered using a handdriven micro-injector (P3 and P7). Doses administered were $10 \mathrm{mg} / \mathrm{kg}$ body weight of ketamine (ketamine hydrochloride), and $10 \mu \mathrm{g} / \mathrm{kg}$ body weight of dexmedetomidine (dexmedetomidine hydrochloride). The control P3 (P3C) and P7 (P7C) pups received no anaesthesia. The treatment pups received ketamine (P3K), ketamine and dexmedetomidine (P3KD), and dexmedetomidine (P3D) respectively. Similar administration was given to P7 pups P7K, P7KD and P7D.

\section{Behavioural testing and assessment of spinal reflex function}

\section{Mechanical withdrawal threshold}

The P3 and P7 rat pups were tested for mechanical withdrawal threshold. The threshold was determined using calibrated von Frey filaments. The von Frey filaments apply logarithmically increasing pressure $(0.4$ to $15 \mathrm{~g})$. Pups were lightly restrained on a flat surface and well calibrated von Frey hairs device (electronic von Frey device, Stoelting, IL, USA) that deliver increasing mechanical stimuli which were applied to the dorsal surface of the hind paw of the pups five times with one second intervals [15]. The number of evoked withdrawal responses to each stimulus of increasing intensity was recorded until a given stimulus evoked five responses or a supra-threshold cut-off pressure was reached [15]. Mechanical withdrawal thresholds were measured in both hind limbs at 15, 30 and 45 min following intrathecal injections.

Separate groups of $P 3$ rat pups $(n=10)$ received intrathecal injections as described above. The pups were maintained under standard conditions as previously described until P35. Spinal cord function was assessed at P35 by evaluating hind limb withdrawal reflex responses to mechanical stimuli [16]. The mechanical stimulus using electronic von Frey device was applied to the mid-plantar surface of the hind paw as previously described under acute withdrawal test. 


\section{Gait analysis at P35}

P35 pups that received intrathecal injections at P3 were assessed for gait. These experiments were performed as described earlier [10,14]. Gait analysis was performed as the animal crossed the glass runway of the CatWalK® system (version 7.1.6) (Noldus Information Technology, Wageningen, The Netherlands). At P22 - 25, the rats were placed on one end of the runway and allowed to explore the environment for about 5 $\min$ for three consecutive days.

The rats were trained, as previously described $[17,18]$ with runway crossings toward feed rewards at the farther end. At P35, runway crossings of the rats were recorded. The values were included for analysis, provided the maximal time for crossing the $60-\mathrm{cm}$-long section of the runway used for gait recording was $\leq 2 \mathrm{sec}$, and when there were no intermediate stops during the crossing. Three crossings per animal were analyzed using the CatWalk $₫$ 7.1.6 software.

\section{Spinal cord tissue preparation and staining}

Tissue analysis was performed $24 \mathrm{~h}$ or 7 days following intrathecal injections. The rat pups that were terminally anesthetized with pentobarbital $(100 \mathrm{mg} / \mathrm{kg}$, i.p) were transcardially perfused with saline followed by $4 \%$ paraformaldehyde. The spinal cord were dissected and fixed in $4 \%$ paraformaldehyde overnight, transferred to $30 \%$ sucrose solution and stored at $4{ }^{\circ} \mathrm{C}$. The transverse sections of lumbosacral spinal cord $(7$ and $14 \mu \mathrm{m}$ ) were excised using a cryostat and fixed on slides and stored at $-30^{\circ} \mathrm{C}$.

\section{Activated caspase- 3 assay}

To assess apoptosis, spinal tissue obtained from P3 and P7 animals $24 \mathrm{~h}$ following intrathecal injection was stained for activated caspase- 3 . The slides were incubated in $3 \%$ peroxidase for $10 \mathrm{~min}$, blocked with $0.3 \%$ Triton X-100 and $5 \%$ normal goat serum in Tris-buffered saline for one hour at room temperature, followed by incubation with rabbit monoclonal anti-activated caspase 3 (1:100; Cell Signalling, Danvers, MA,USA) overnight at $4{ }^{\circ} \mathrm{C}$. Biotinylated goat anti-rabbit secondary antibody (Vector Laboratories, Burlingame, CA, USA) was applied at 1:250 for $30 \mathrm{~min}$ at room temperature. The slides were further incubated with avidin-biotin-peroxidase complex (Vector Laboratories, Burlingame, CA) for $30 \mathrm{~min}$ and developed with 3,3'diaminobenzidine (DAB) for $8 \mathrm{~min}$. The slides were counterstained with hematoxylin, dehydrated and observed.

\section{Fluoro-Jade C staining}

Fluoro-Jade C staining was performed in $14 \mu \mathrm{m}$ sections of spinal cord obtained $24 \mathrm{~h}$ after injection. The staining was done using FluoroJade $\mathrm{C}$ staining kit (Biosensis, USA) following the manufacturer's instructions. Fluoro-Jade C immunfluorescent positive cells were counted under the fluorescent microscopy.

\section{Evaluation of ionized calcium binding adapter molecule 1 (Iba1)}

Spinal cord sections (14 $\mu \mathrm{m}$ thick) were evaluated 7 days following injection for glial reactivity with microglial (Iba1) marker. The slides after washing with Triton X-100 $(0.1 \%$ in phosphate buffered saline), were incubated in 5 $\%$ goat blocking serum at room temperature for 60 min, followed by incubation with rabbit antiIba1 (1:1000; WAKO Chem, Irvine, CA, USA) for $48 \mathrm{~h}$ at $4{ }^{\circ} \mathrm{C}$. The slides were further incubated with fluorescent secondary antibodies for $2 \mathrm{~h}$ (1:250 goat anti-mouse Alexa 555 and 1:250 goat anti-rabbit Alexa 488; Abcam, USA). Slides were cover-slipped with prolong gold antifade mounting media with 4',6-diamidino-2phenylindole (DAPI). The sections were imaged using an Olympus BX51 (Olympus America, Inc., Center Valley, PA, USA) equipped with a digital camera and image-capture software. The mean intensity of immunofluorescence was calculated using Image Pro Plus software (Media Cybernatics Inc., Silver Spring, MD) [26].

\section{Statistical analysis}

The data are presented as mean \pm SD and were analysed using SPSS version 17.0 software. One way ANOVA $(p<0.05)$ followed by DMRT analysis was done.

\section{RESULTS}

\section{Mechanical withdrawal threshold}

The mechanical withdrawal thresholds following intrathecal ketamine were observed to be markedly lower in P3 and P7 at 15 and 30 min when compared to control pups (Figure 1).

The threshold values were lower at $45 \mathrm{~min}$ however the values did not show a significant change as compared to control pups. The threshold levels of P3KD and P7KD were observed to be slightly higher than the ketamine treated P3K and P7K pups (Fig 2). Intrathecal dexmedetomidine at $\mathrm{P} 3$ and $\mathrm{P} 7$ presented 
A

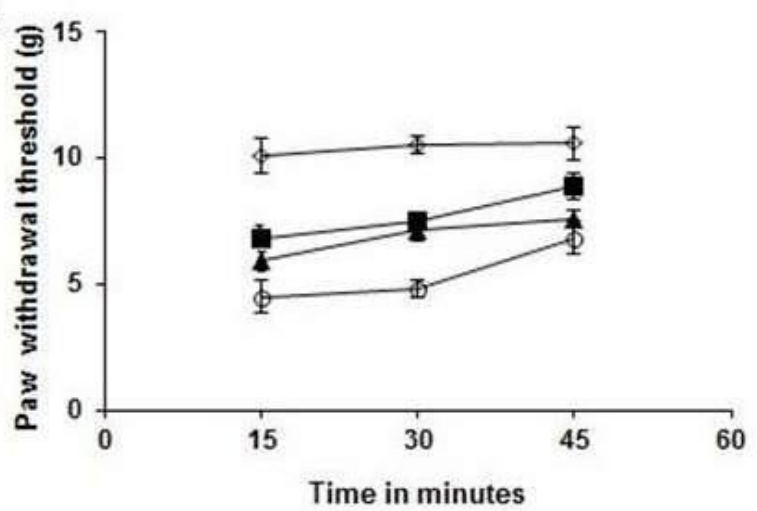

B

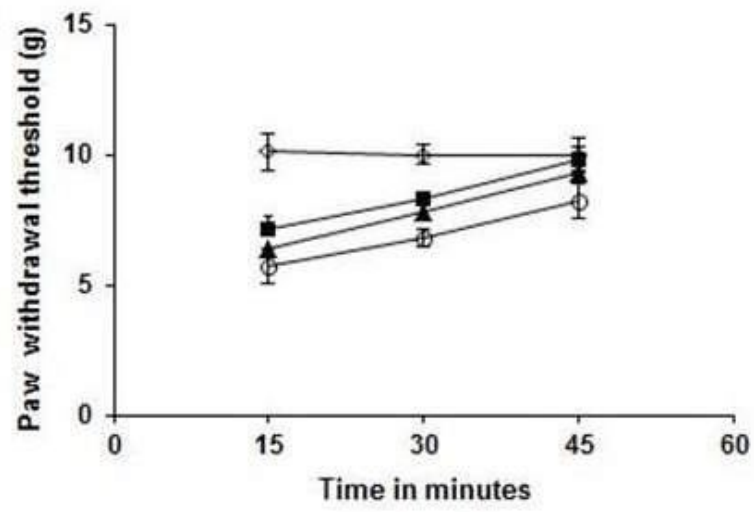

Figure 1: Mechanical withdrawal thresholds (determined by von Frey Filaments) of P3 (A) and P7 (B) rat pups at 15,30 and 45 min following intrathecal injections. Values are represented as mean \pm SD $(n=6) ; \quad$ Symbol key: $\diamond=$ control; o = ketamine; $\boldsymbol{\Lambda}$ = ketamine+dexmedetomidine; $\boldsymbol{\|}=$ dexmedetomidine

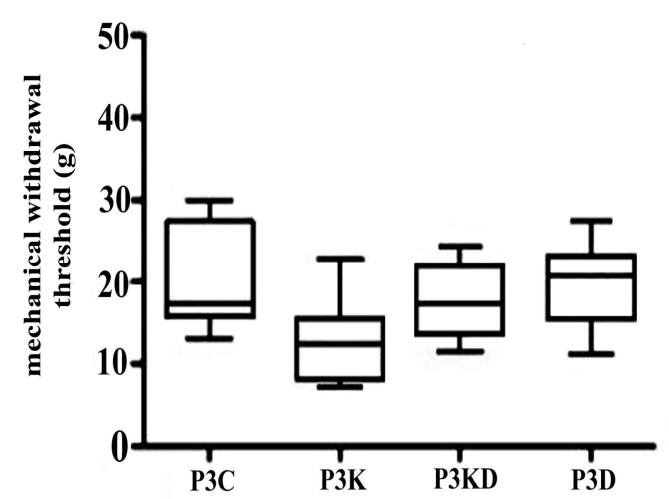

Figure 2: Mechanical withdrawal thresholds (determined by von Frey Filaments) of at P35 pups that received intrathecal injections on P3. Values are represented as mean $\pm \mathrm{SD}$

mechanical threshold levels almost similar to P3C and P7C, except for at $15 \mathrm{~min}$ where the values were less compared to P3C and P7C. Separate groups of rat pups receiving ketamine at P3 were evaluated at P35. At P35, mechanical withdrawal thresholds were lower in the P3 ketamine group compared with the $\mathrm{P} 3 \mathrm{C}$ group $(p$
$<$ 0.05). However, groups that received dexmedetomidine alone and ketamine + dexmedetomidine showed threshold values that differed non-significantly against control pups that received neither dexmedetomidine nor ketamine.

\section{Gait analysis}

Gait analysis was performed using the CatWalk® runway system at P35. The system records both static and dynamic parameters such as paw pressure, print area, duty cycle, stride length as well as interlimb coordination. Ketamine injections at P3 resulted in a marked $(p<0.05)$ decrease in paw print area and paw print intensity. However the decreases in dynamic parameters were not significant (Table 1). The treatment with intrathecal injections of ketamine + dexmedetomidine and dexmedetomidine did not result in considerable alterations in gait as compared to the control pups.

Table 1: Gait parameters for rat pups exposed to anaesthesia

\begin{tabular}{lcccccc}
\hline \multirow{2}{*}{ Group } & \multicolumn{2}{c}{ Static parameter } & \multicolumn{2}{c}{ Dynamic parameter } \\
\cline { 2 - 6 } & $\begin{array}{c}\text { Paw print } \\
\text { area }\left(\mathbf{m m}^{2}\right)\end{array}$ & $\begin{array}{c}\text { Paw print } \\
\text { intensity }\end{array}$ & $\begin{array}{c}\text { Regulatory } \\
\text { index }\end{array}$ & Duty cycle & $\begin{array}{c}\text { Stride length } \\
(\mathbf{c m})\end{array}$ & $\begin{array}{c}\text { Stability of } \\
\text { gait (mm) }\end{array}$ \\
\hline P3C & $43.10 \pm 1.06^{\mathrm{b}}$ & $170.20 \pm 10.10^{\mathrm{C}}$ & $99.9 \pm 0.16^{\mathrm{a}}$ & $59.25 \pm 1.14^{\mathrm{a}}$ & $109.70 \pm 9.36^{\mathrm{a}}$ & $32.9 \pm 1.21^{\mathrm{a}}$ \\
P3K & $20.25 \pm 0.68^{\mathrm{a}}$ & $120.25 \pm 9.15^{\mathrm{a}}$ & $95 \pm 0.69^{\mathrm{a}}$ & $54.35 \pm 1.38^{\mathrm{a}}$ & $100.10 \pm 8.65^{\mathrm{a}}$ & $27.10 \pm 1.01^{\mathrm{a}}$ \\
P3KD & $38.50 \pm 2.01^{\mathrm{b}}$ & $158.80 \pm 11.21^{\mathrm{b}}$ & $98 \pm 0.53^{\mathrm{a}}$ & $56.20 \pm 2.05^{\mathrm{a}}$ & $109.16 \pm 9.81^{\mathrm{a}}$ & $33.15 \pm 1.00^{\mathrm{a}}$ \\
P3D & $39.10 \pm 0.56^{\mathrm{b}}$ & $160.10 \pm 7.36^{\mathrm{b}}$ & $99 \pm 0.25^{\mathrm{a}}$ & $58.75 \pm 2.10^{\mathrm{a}}$ & $108.25 \pm 3.45^{\mathrm{a}}$ & $33.80 \pm 0.96^{\mathrm{a}}$ \\
\hline
\end{tabular}

Values are represented as mean \pm SD $(n=10)$; values within the same column that share a common alphabet do not differ significantly (ANOVA, $p<0.05$ ) followed by DMRT analysis; Paw Print area = surface area of floor contacted by hindpaw; Paw Print intensity = intensity of pixels forming area of paw contact; Regularity index = index for degree of interlimb coordination during gait; Stability of gait = distance between two hindpaws measured perpendicular to walking direction; Stride length = distance between placement of hindpaw and subsequent placement of same paw; Duty cycle = ratio between stance duration and full stepcycle duration 


\section{Activated caspase-3}

Activated caspase- 3 was detected in the spinal tissue sections to identify apoptotic neurons. The number of apoptotic cell profiles was significantly $(p<0.05)$ increased (Figure 3$) 24 \mathrm{~h}$ subsequent to intrathecal ketamine of $10 \mathrm{mg} / \mathrm{kg}$, in P3K and in P7K pups $(p<0.05)$.

In P3KD and P7KD animals, apoptotic cell count increases following intrathecal injections of ketamine and dexmedetomidine were not statistically significant when compared with respective control pups. The increase was slightly lesser in P7 pups when compared to P3 pups. The rise in apoptotic cells in intrathecal dexmedetomidine administration in P3 and P7 pups (P3D and P7D) was not significant.

\section{Fluoro-Jade C}

The number of Fluoro-Jade $C$ positive cells in spinal cord sections of the rat pups at P3 and P7 following $24 \mathrm{~h}$ after injections are represented in Figure 4 . The results reveal a significant $(p<$ $0.05)$ increase in the Fluoro-Jade $C$ positive cell counts $24 \mathrm{~h}$ after injections of $10 \mathrm{mg} / \mathrm{kg}$ ketamine in the P3K pups as compared to control P3 pups. The number was slightly reduced in the P3KD pups and significantly less in the P3D pups as against P3K pups treated with ketamine alone. Following ketamine at P7 there was a slight increase in positive cell counts in P7K as against P7C that was not statistically significant. FluoroJade $C$ positive cells were rarely observed in the P7KD and in P7D pups, however the counts were higher than in P7C.

(A)
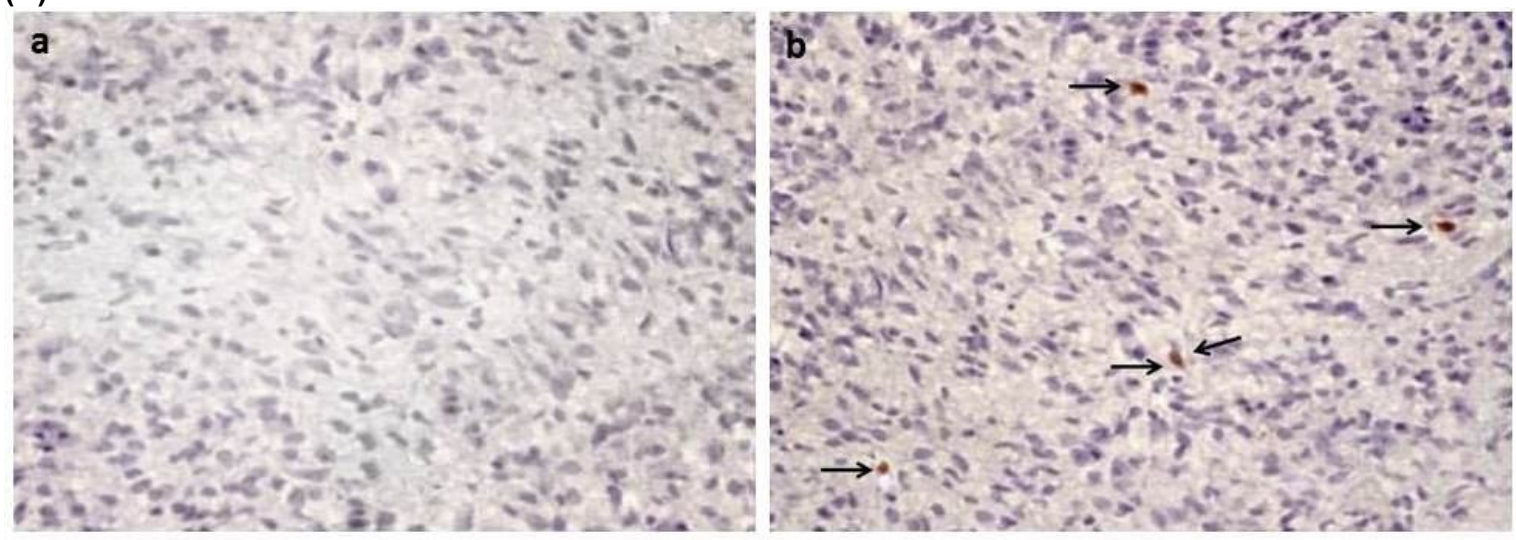

(B)

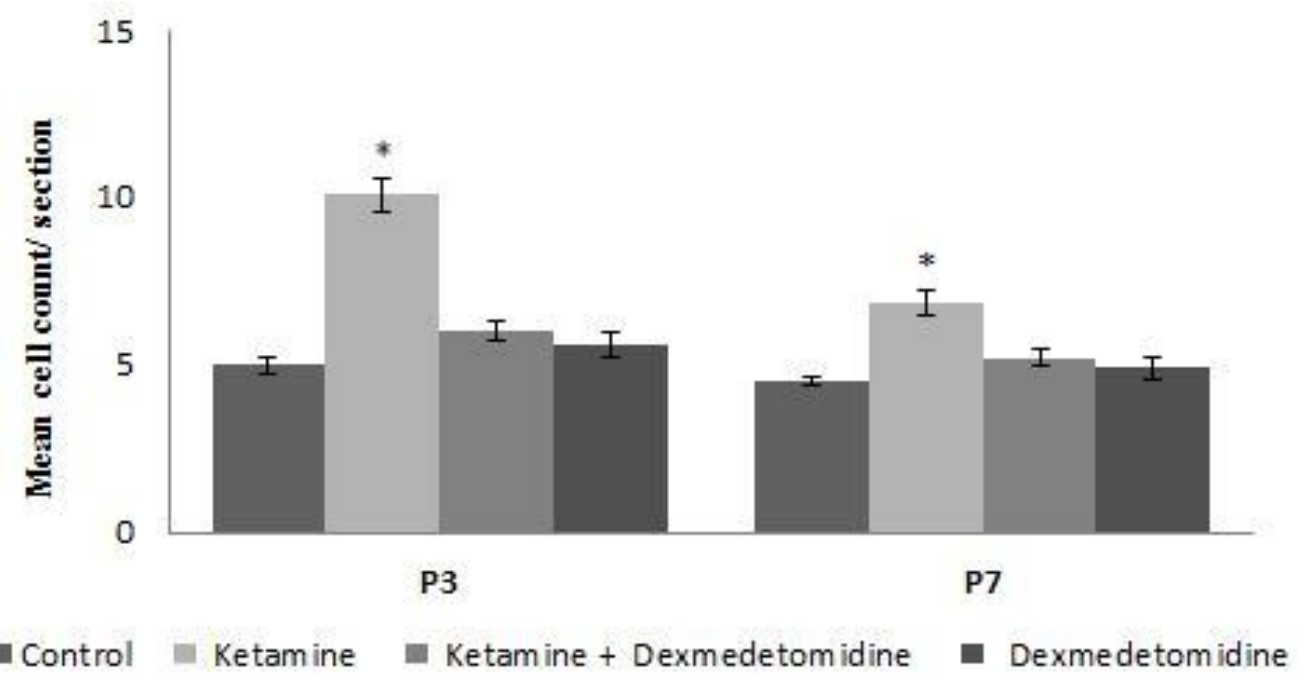

Figure 3: Activated caspase-3 immunostaining following intrathecal injections in P3 and P7 rats pups; (A) Representative sections of spinal cord (a) Control; (b) $24 \mathrm{~h}$ after injections; (B) Activated caspase-3 immunopositive cell counts $24 \mathrm{~h}$ after intrathecal injections; values represented as mean $\pm \mathrm{SD}(\mathrm{n}=3)$. * represents values that vary significantly at $p<0.05$ 


\section{Glial reactivity}

lonized calcium binding adapter molecule 1 (Iba1)

Glial reactivity was assessed by detecting ionized calcium binding adapter molecule 1 (Iba1). Iba1 immunoreactivity in the spinal cord increased 7 days following injection of intrathecal ketamine $10 \mathrm{mg} / \mathrm{kg}$ in P3 pups (Figure 5). No significant change was observed following ketamine injection in P7 pups as well in P3KD and P7KD pups. Intrathecal dexmedetomidine administration also resulted in no significant change in Iba1 as compared to P3C and P7C pups.

\section{DISCUSSION}

Ketamine, a non-competitive NMDA receptor antagonist is a widely used dissociative anaesthetic agent, and blockade of NMDA receptors is known to cause neurotoxicity $[7,19]$. In the present study, the influence of dexmedetomidine administration on the effect of intrathecal ketamine in neonatal rat pups was evaluated. The spinal function was assessed by mechanical withdrawal threshold and gait parameters at P35. The hind paw withdrawal threshold was determined using von Frey hairs and was expressed in grams. The animal's response to these filaments was used to determine mechanical withdrawal thresholds. Usually, a paw withdrawal upon probing or immediately upon release of the filament is considered a positive response [20]. The results of the study revealed the spinal toxicity of intrathecal ketamine administration at P3 and P7, as evidenced by a lower threshold levels that improved on administration of dexmedetomidine.

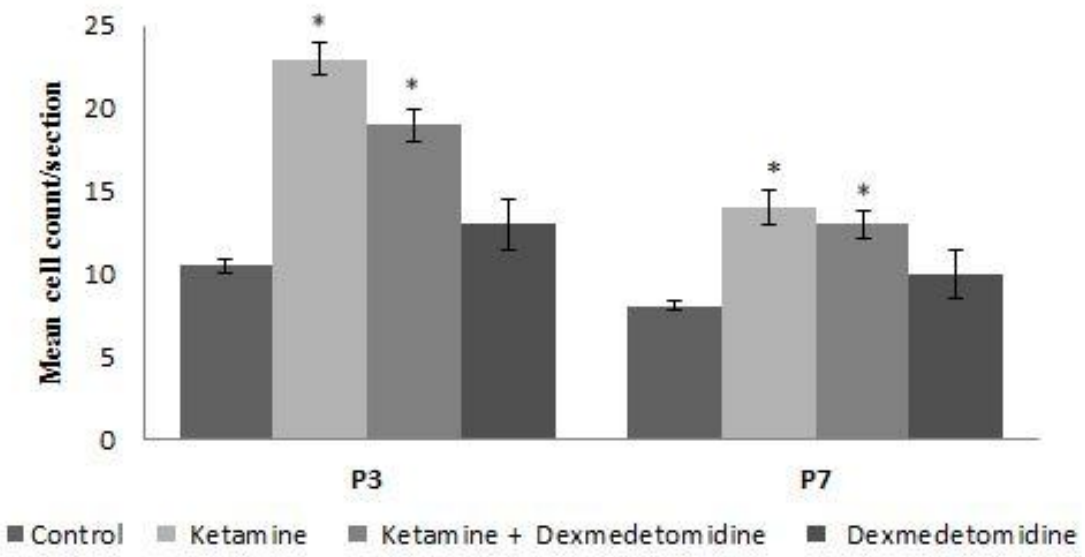

Figure 4: Apoptopic cell counts by Fluoro-Jade $C$ staining. Apoptotic cell counts in spinal cord sections of P3 rat pups after intrathecal injections; values presented as mean \pm SD $(n=3)$; ${ }^{*}$ represents values that vary significantly $(p<0.05)$

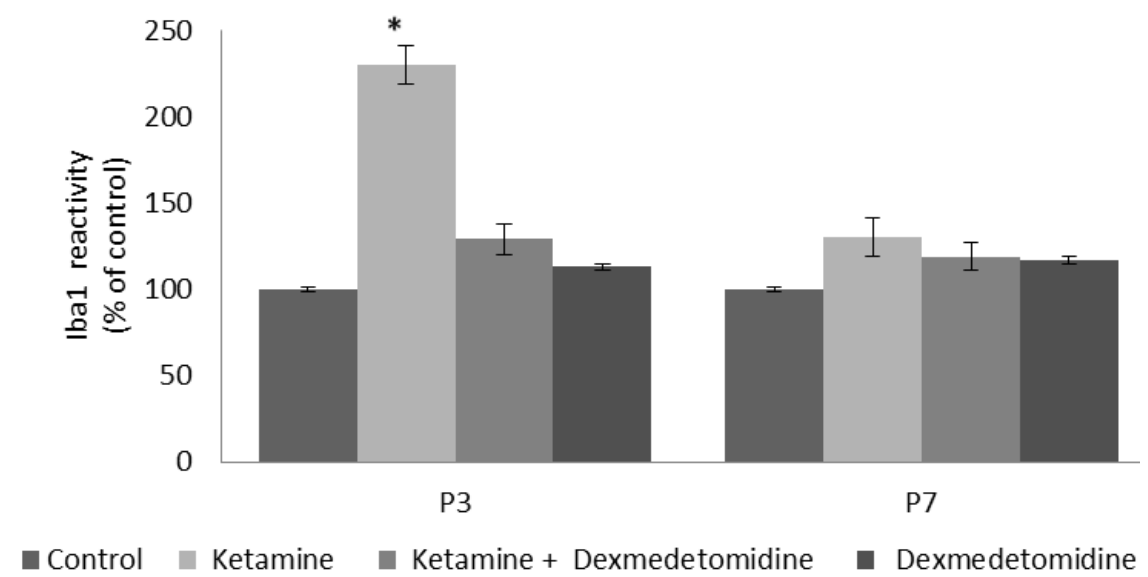

Figure 5: Ionized calcium binding adapter molecule 1 (Iba1) immunoreactivity. Immunofluorescence presented as $\%$ change in positive area fraction compared with control animals; values are presented as mean $\pm \operatorname{SD}(n=3)$; * represents values that vary significantly $(p<0.05)$ 
Following intrathecal ketamine at P3, mechanical withdrawal threshold was reduced at P35. Likewise, NMDA antagonist MK-801 placed over the spinal cord of $\mathrm{P}$ 0-1 pups as a slow release formula [21] revealed persistent changes in mechanical sensitivity, and the level of change was greater with extended blockade.

Local anaesthetics may have other toxic effects that are not related to developmental apoptosis and that which influence both motor and sensory outcomes. Recent studies have demonstrated that local anaesthetic causes changes in thermal tail flick latency, mechanical paw pressure withdrawal threshold and motor function 4 to 7 days following doses of local anaesthetic [21]. The gait analysis of experimental animals was performed by the CatWalk system, which is a video-based automated gait analysis system that evaluates gait changes in rodents [22]. Intrathecal ketamine injection resulted in alterations in the gait parameters. Hind paw print area and print intensity decreased following intrathecal ketamine injections at P3, suggesting impairments in placement of the paw on the glass during the static phase of walking. Walker et al [10] also reported a similar finding. Marked decreases in hind paw print area and print intensity evocative of allodynia have been observed to be associated with pain as in monoarthritis [23], and in conditions of peripheral nerve lesions [18]. Subsequent to P3 ketamine administration, duty cycle was decreased, because the time hind paw was in contact with the surface during gait was decreased. However, the differences were not statistically significant. The results of dynamic parameters - stride length, gait regularity and stability suggested that gait coordination was slightly decreased as against control pups. In animals that received intrathecal ketamine and dexmedetomidine, there was a marked improvement in gait. The static parameters were increased markedly as against pups that received intrathecal ketamine alone. Similar results were observed in dexmedetomidine alone administered rat pups. The print area and print intensity of the pups were almost near control values. Intrathecal dexmedetomidine could possibly offer protection against ketamine-induced alterations in gait.

The developing spinal cord be susceptible to apoptosis, which increases following prolonged general anaesthesia at P7 [24] and as well to analgesic doses of intrathecal ketamine at P3 [10]. Although apoptosis has been reported in spinal cord following anaesthesia at P7 [21], the period of peak susceptibility to spinal toxicity differs. It has been reported that the degree of developmental apoptosis in the lumbar spinal cord changes with age [25]. In the present study, the results obtained indicate that the level of apoptosis was markedly greater than for P7 pups. Activated caspase-3, an enzyme in the apoptotic cascade, identifies neurons that have progressed beyond the point of commitment to cell death [26]. Antibodies to activated caspase-3 have been employed to assess neuronal apoptosis and Fluoro-Jade $\mathrm{C}$, a sensitive marker of neuronal degeneration [10] was used to determine cellular degeneration. In the spinal cord, the number of apoptotic profiles was increased by 2 folds following intrathecal ketamine in line with previous reports [10]. Administration of dexmedetomidine alone and with ketamine resulted in lesser apoptotic cell counts as measured by caspase- 3 and FluoroJade $\mathrm{C}$ staining.

Neuronal apoptosis was increased by intrathecal ketamine along with increased microglial activation 7 days following ketamine injection and changes in sensory threshold and gait, several weeks later. Ionized calcium binding adaptor molecule 1 (lba1) is a microglia/macrophagespecific calcium-binding protein. Iba1 participates in membrane ruffling and phagocytosis in activated microglia. Previous studies have shown activation of microglia commonly occurring in the early response of the CNS to a wide variety of pathological stimuli, including axotomy, trauma, inflammation, degeneration, and ischemia [27]. Several studies also have suggested that microglia may be rapidly and time-dependently activated after ischemia, and the activation may reflect the extent of severity of ischemic injury [28]. Iba1 thus serves as a marker in pathological conditions. Microglia has been known to exert dual effects on the CNS [27]. It is involved in neuro-protection by secretion of several neurotrophins and elimination of microorganisms and deleterious debris [29]. In contrast, microglia also exerts neurotoxic effects by releasing reactive oxygen species, nitric oxide, or inflammatory cytokines, which may cause neuronal damage [30].

In the present study, the marked increase in Iba1 in the ketamine treated pups is suggestive of the microglial activation. This could be either due to its neuro-protective or neurotoxic effects under the influence of ketamine. Nevertheless, the considerable decrease in the level of lba1 in the pups treated with dexmedetomidine either alone or along with ketamine is suggestive of the protective effect of dexmedetomidine. Studies have shown the neuro-protective properties of dexmedetomidine [31]. These data suggested that dexmedetomidine might be helpful in 
reducing the neurotoxicity induced by local anaesthetics.

\section{CONCLUSION}

The observed results of mechanical withdrawal and gait analysis are in line with neuronal apoptotic cell counts. Thus, dexmedetomidine offers considerable neuro-protection against intrathecal ketamine.

\section{REFERENCES}

1. Kokki H. Spinal blocks. Paediatr Anaesth 2012; 22: 5664.

2. Walker SM, Yaksh TL. Neuraxial analgesia in neonates and infants: a review of clinical and preclinical strategies for the development of safety and efficacy data. Anesth Analg 2012; 115: 638 - 662.

3. Kokki H, Tuovinen K, Hendolin H. Spinal anaesthesia for paediatric day-case surgery: $A$ doubleblind, randomized, parallel group, prospective comparison of isobaric and hyperbaric bupivacaine. $\mathrm{Br} J$ Anaesth 1998; 81: 502-506.

4. Brouwers $M$, Driessen J, Severijnen R. Clinical letter: Epidural analgesia in a newborn with Hirschsprung's disease, associated with congenital central hypoventilation syndrome. Eur J Anaesthesiol 2000; 17: 751-753.

5. Mellon RD, Simone AF, Rappaport BA. Use of anesthetic agents in neonates and young children. Anesth Analg 2007; 104: 509-520.

6. Haley-Andrews S. Ketamine: the sedative of choice in a busy pediatric emergency department. J Emerg Nurs 2006; 32: 186-188.

7. Ikonomidou C, Bosch C, Miksa M, Bittigau P, Vockler J, Dikranian K, Tenkova TI, Stefovska V, Turski L, Olney JW. Blockade of NMDA receptors and apoptotic neurodegeneration in the developing brain. Science 1999; 283: 70-74.

8. Ansermino $M$, Basu $R$, Vandebeek $C$, Montgomery $C$. Nonopioid additives to local anaesthetics for cauda blockade in children: A systematic review. Paediatr Anaesth 2003; 13: 561-573.

9. Eisenach JC, Yaksh TL. Epidural ketamine in healthy children: What's the point? (letter). Anesth Analg 2003; 96: 626.

10. Walker SM, Westin BD, Deumens R, Grafe M, Yaksh TL. Effects of intrathecal ketamine in the neonatal rat: evaluation of apoptosis and long-term functional outcome. Anesthesiology 2010; 113: 147-159.

11. Ma D, Hossain $M$, Rajakumaraswamy $N$, Arshad $M$, Sanders RD, Franks NP, Maze M. Dexmedetomidine produces its neuroprotective effect via the $\alpha 2 A$ adrenoceptor subtype. Eur J Pharmacol 2004; 502. 87-97.
12. Levanen J, Makela ML, Scheinin H. Dexmedetomidine premedication attenuates ketamine-induced cardiostimulatory effects and postanesthetic delirium. Anesthesiology 1995; 82: 1117-1125.

13. Committee for the Update of the Guide for the Care and Use of Laboratory Animals. Guide for the care and use of laboratory animals, 8th edn. The National Academies Press, Washington, DC, 2011.

14. Westin BD, Walker SM, Deumens R, Grafe M, Yaksh TL. Validation of a preclinical spinal safety model: Effects of intrathecal morphine in the neonatal rat. Anesthesiology 2010; 113: 183-199.

15. Walker SM, Howard RF, Keay KA, Fitzgerald M. Developmental age influences the effect of epidural dexmedetomidine on inflammatory hyperalgesia in rat pups. Anesthesiology 2005; 102: 1226-1234.

16. Walker SM, Grafe M, Yaksh TL. Intrathecal clonidine in the neonatal rat: Dose-dependent analgesia and evaluation of spinal apoptosis and toxicity. Anesth Analg 2012; 115: 450-460.

17. Deumens R, Koopmans GC, Honig WM, Hamers FP, Maquet $V$, Jerome $R$, Steinbusch HW, Joosten EA. Olfactory ensheathing cells, olfactory nerve fibroblasts and biomatrices to promote long-distance axon regrowth and functional recovery in the dorsally hemisected adult rat spinal cord. Exp Neurol 2006; 200: 89-103.

18. Deumens $R$, Jaken $R J$, Marcus MA, Joosten EA. The CatWalk gait analysis in assessment of both dynamic and static gait changes after adult rat sciatic nerve resection. J Neurosci Methods 2007; 164: 120-130.

19. Wang C, Sadovova N, Fu X, Schmued L, Scallet A, Hanig J, Slikker $W$. The role of the N-methyl-Daspartate receptor in ketamine-induced apoptosis in rat forebrain culture. Neuroscience 2005; 132: 967977.

20. Chaplan SR, Bach FW, Pogrel JW, Chung JM, Yaksh TL. Quantitative assessment of tactile allodynia in the rat paw. J Neurosci Methods 1994; 53: 55-63.

21. Beggs S, Torsney C, Drew LJ, Fitzgerald M. The postnatal reorganization of primary afferent input and dorsal horn cell receptive fields in the rat spinal cord is an activity-dependent process. Eur $J$ Neurosci 2002; 16: 1249-1258.

22. Hamers FP, Lankhorst AJ, van Laar TJ, Veldhuis WB, Gispen WH. Automated quantitative gait analysis during overground locomotion in the rat: its application to spinal cord contusion and transection injuries. J Neurotrauma 2001; 18: 187-201.

23. Angeby-Moller K, Berge OG, Hamers FP. Using the CatWalk method to assess weight-bearing and pain behaviour in walking rats with ankle joint monoarthritis induced by carrageenan: Effects of morphine and rofecoxib. J Neurosci Methods 2008; 174: $1-9$.

24. Sanders RD, $X u J$, Shu $Y$, Fidalgo A, Ma D, Maze $M$. General anesthetics induce apoptotic 
neurodegeneration in the neonatal rat spinal cord. Anesth Analg 2008; 106: 1708-1711.

25. Lowrie MB, Lawson SJ. Cell death of spinal interneurones. Prog Neurobiol 2000; 61: 543-555.

26. Jevtovic-Todorovic V, Olney JW. PRO: Anesthesiainduced developmental neuroapoptosis: Status of the evidence. Anesth Analg 2008; 106: 1659-1663.

27. Moore $S$, Thanos $S$. The concept of microglia in relation to central nervous system disease and regeneration. Prog Neurobiol 1996; 48: 441-460.

28. Kato $H$, Kogure $K$, Liu XH, Araki T, Itoyama $Y$. Progressive expression of immunomolecules on activated microglia and invading leukocytes following focal cerebral ischemia in the rat. Brain Res 1996, 734: 203-212.
29. Hamanoue M, Takemoto N, Matsumoto K, Nakamura T, Nakajima K, Kohsaka S. Neurotrophic effect of hepatocyte growth factor on central nervous system neurons in vitro. J Neurosci Res 1996; 43: 554-564.

30. Smith ME, van der Maesen K, Somera FP. Macrophage and microglial responses to cytokines in vitro: phagocytic activity, proteolytic enzyme release, and free radical production. J Neurosci Res 1998; 54: 6878.

31. Bruzzone A, Pinero CP, Castillo LF, Sarappa MG, Rojas $P$, Lanari C, Luthy IA. Alpha2-adrenoceptor action on cell proliferation and mammary tumour growth in mice. Br J Pharmacol 2008; 155: 494-504. 